\title{
Higher than expected carrier rates for familial Mediterranean fever in various Jewish ethnic groups
}

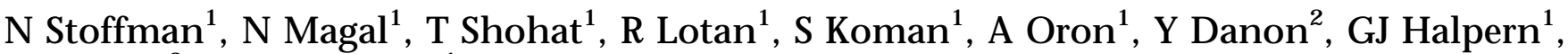 \\ $Y$ Lifshitz $^{3}$ and $M$ Shohat $^{1}$
}

\begin{abstract}
${ }^{1}$ Department of Medical Genetics, FMRC, Schneider Children's Medical Center of Israel, and Rabin Medical Center; ${ }^{2}$ Kipper Institute of Immunology, Schneider Children's Medical Center of Israel; ${ }^{3} \mathrm{M}$ aternal Health Center, Kupat Holim Klalit, Ramla, and Sackler School of Medicine, Tel Aviv University, Israel
\end{abstract}

Familial Mediterranean fever (FMF) is an autosomal recessive disease characterised by recurrent attacks of inflammation of serosal membranes. Amyloidosis leading to renal failure is the most severe complication in untreated patients. In Israel FMF is most frequent among Jews of North African origin. Recently the causative gene (MEFV) has been found and the common mutations characterised. The aim of this study was to investigate the carrier rates of the common MEFV mutations among $\mathbf{4 0 0}$ healthy members of four different ethnic groups (100 in each group) in Israel, and to compare the distribution of the different mutations between FMF carriers and patients. We found a high frequency of carriers among Jews from the various ethnic groups. In North African Jews it was $22 \%$, in Iraqi Jews $39 \%$, in Ashkenazi Jews $21 \%$, and in Iranian Jews $6 \%$. The distribution of the four most common MEFV mutations among healthy individuals (M694V 29\%, V726A 16\%, M680I 2\% and E148Q 53\%) was significantly different $(P<0.003)$ from that found in patients (M694V 84.4\%, V726A 9.0\%, M680I 0\% and E148Q 6.6\%). Six healthy asymptomatic individuals were found to carry mutations in both alleles: two homozygotes for E148Q and four compound heterozygotes $\mathrm{E} 148 \mathrm{Q} /$ other. These results demonstrate a very high carrier rate among all Jewish ethnic groups. They confirm that mutation E148Q is associated with a milder phenotype, which explains the lower prevalence of FMF among the Ashkenazi and Iraqi Jews. This study raises the question of the need for molecular screening for M694V homozygotes in the Israeli North African Jewish community. European Journal of Human Genetics (2000) 8, 307-310.

Keywords: FMF; mutations; carrier rate; Jews; Israel

\section{Introduction}

Familial Mediterranean fever (FMF) is characterised by recurrent short episodes of inflammation and serositis including fever, peritonitis, pleuritis, and synovitis. ${ }^{1}$ Colchicine has been shown to be effective in preventing the attacks of FMF as well as the development of amyloidosis. ${ }^{2}$

The gene causing FMF (MEFV) has been cloned, ${ }^{3,4}$ and three common founder MEFV mutations were initially found

Correspondence: Mordechai Shohat, MD, Director, Department of Medical Genetics, Rabin Medical Center, Beilinson Campus, Petah Tikva, 49100, Israel. Tel: +9723937 7658/9; Fax: +9723937 7660; E-mail: mshohat@ccsg.tau.ac.il

Received 21 June 1999; revised 21 October 1999; accepted 2 November 1999 in exon 10: M694V, M680I and V726A. ${ }^{3,4}$ Additional less common mutations have been found subsequently in exons 10, 3 and 5, and more recently a common mutation has been found in exon 2: E148Q. ${ }^{5}$ Genotype/phenotype correlation has demonstrated higher morbidity with mutation M694V, especially with regard to the risk of developing amyloidosis. ${ }^{6-11}$ Based on prevalence patterns, it is clear that this disease is common in the Mediterranean area, mainly among Armenians, Turks, Arabs and Jews. In Israel it has been shown that $53-61 \%$ of all the patients are of North African origin. A high percentage (16-24\%) of Jews of Iraqi and Kurdish origin are also affected, while $10 \%$ of patients are Ashkenazi Jews. ${ }^{12,13}$ Recently Aksentijevich et $\mathrm{al}^{14}$ found a carrier rate for MEFV mutations of $21 \%$ in 200 anonymous Ashkenazi Jewish DNA samples with E148Q being the most 
common mutation. This further suggests a heterozygote advantage in the Mediterranean area.

The aim of our study was to find the carrier rates for the different mutations in the MEFV gene in different Jewish ethnic groups in Israel. Since amyloidosis correlates with homozygosity for M 694V, ${ }^{6-11}$ and since this complication can bethe first and only manifestation of FM F with no inflammatory attacks (phenotype2), ${ }^{15}$ such information could help in determining the need for mutation detection screening in the different ethnic groups in the general population.

\section{Subjects and methods}

We studied 400 blood samples from healthy individuals who were referred for cystic fibrosis screening belonging to four different ethnic origins - North African Jews (mainly M oroccan), Iraqi Jews, Iranian Jews, and Ashkenazi Jews. The subjects were pooled from the various prenatal clinics of our genetic institute. The blood samples were tested for the four most frequent FMF mutations, three of which are located on exon 10 (M694V, V726A, M680I), and one on exon 2 (E148Q).

Mutation analysis was performed by genomic sequencing. Seven mutations in exon 10 were tested by PCR amplification using the forward oligonucleotide 10F1; 5'-ccagaagaactaccctgtccc-3' and the reverse oligonucleotide 10R1; 5'-cagagcagctggcgaatgtat-3'. The PCR amplification was performed under the following conditions: $95^{\circ} \mathrm{C}$ for $10 \mathrm{~min}$ followed by 30 cycles of $95^{\circ} \mathrm{C}$ for $15 \mathrm{~s}, 55^{\circ} \mathrm{C}$ for $30 \mathrm{~s}$ and $72^{\circ} \mathrm{C}$ for $30 \mathrm{~s}$; and final extension at $72^{\circ} \mathrm{C}$ for $10 \mathrm{~min}$. PCR products were purified with High Pure PCR Product Purification Kit (Boehringer, Mannheim, Germany) and sequenced directly, using specific primers and Thermo Sequenase Kit (Amersham, Buckinghamshire, UK).

Mutation E148Q in exon 2 was analysed by restriction of PCR products from genomic DNA. The region harbouring mutation E148Q was amplified using the forward oligonucleotide: 5'-gcctgaagactccagaccaccccg-3' and the reverse oli- gonucleotide 5'-aggccctccgaggccttctct-3'. The amplified products were digested for $3 \mathrm{~h}$ with $3 \mathrm{IU}$ of BstN 1 .

The frequencies and distribution of the mutationsfound in the carriers were compared with the frequencies and distribution of the mutations found in Israeli FMF probands who were referred randomly to our clinic for confirmation of the clinical diagnosis of FMF. Included in the study were 169 patients of only four ethnic groups: North African Jews (120), Iraqi Jews (20), other non-Ashkenazi Jews (Iran and Levant) (12) and Ashkenazi Jews (17). We considered as FMF patients those found to be homozygotes or compound heterozygotes for the four FMF mutations described above, and patients with diagnostic clinical signs of FMF but molecular findings of only one of the mutations which has been identified up to now. Not all the individuals classified as patients met one of the published sets of clinical criteria for the diagnosis of FMF, but there were some individuals with borderline histories who turned out to be mutation positive. We did not include any individuals with clinical signs of FMF but in whom none of the four mutations was found.

All computations were made with the use of the SAS statistical package. The frequencies of the four most common mutations were calculated with $95 \%$ confidence interval, and the comparison of the distribution of the MEFV mutations between FMF patients and FMF carriers was done by the $\chi^{2}$ test.

This study was approved by the Human Subjects Committee at the Rabin Medical Center, Beilinson Campus, Petah Tikva, Israel, and informed consent was obtained from each participant.

\section{Results}

Table1 depicts the frequency of the four most common mutations of the MEFV gene in the different ethnic groups

Table 1 Frequency of the four most common MEFV mutations (95\% confidence interval) among healthy individuals according to ethnic group

\begin{tabular}{lllllll}
\hline & M 694V & V726A & M 680I & E148Q & M utation frequency & Carrier rate \\
\hline North African & $16 / 200$ & $2 / 200$ & 0 & $10 / 200$ & $28 / 200$ & $22 / 100$ \\
Jews $(n=200)^{\mathrm{a}}$ & $(0.05-0.13)$ & $(0.00-0.03)$ & $(0-0.02)$ & $(0.02-0.09)$ & $(0.09-0.19)$ & $(0.14-0.31)$ \\
Iraqi Jews & $11 / 200$ & $10 / 200$ & $1 / 200$ & $23 / 200$ & $45 / 200$ & $39 / 100$ \\
$(\mathrm{n}=200)^{\mathrm{b}}$ & $(0.03-0.10)$ & $(0.02-0.09)$ & $(0.0001-0.03)$ & $(0.07-0.17)$ & $(0.17-0.29)$ & $(0.29-0.49)$ \\
Iranian Jews & $1 / 200$ & 0 & 0 & $5 / 200$ & $6 / 200$ & $6 / 100$ \\
$(\mathrm{n}=200)$ & $(0.00-0.03)$ & $(0.00-0.02)$ & $(0.00-0.02)$ & $(0.01-0.06)$ & $(0.01-0.06)$ & $(0.02-0.13)$ \\
Ashkenazi Jews & $1 / 200$ & $4 / 200$ & $1 / 200$ & $15 / 200$ & $21 / 200$ & $21 / 100$ \\
$(\mathrm{n}=200)^{\mathrm{c}}$ & $(0.00-0.03)$ & $(0.01-0.05)$ & $(0.0001-0.03)$ & $(0.04-0.08)$ & $(0.07-0.16)$ & $(0.13-0.30)$ \\
Total $(n=800)$ & $29 / 800$ & $16 / 800$ & $2 / 800$ & $53 / 800$ & $100 / 800$ & $88 / 400$ \\
& $(0.024-0.052)$ & $(0.011-0.03)$ & $(0.00-0.01)$ & $(0.05-0.86)$ & $(0.10-0.15)$ & $(0.18-0.26)$ \\
\hline
\end{tabular}

an this group was one individual who was homozygous for E148Q and two others who were compound heterozygotes E148Q/M 694V; ${ }^{b}$ In this group was one individual who was homozygous for E148Q and two others who were compound heterozygotes: one E148Q/M 694V and one $\mathrm{E} 148 \mathrm{Q} / \mathrm{V} 726 \mathrm{~A}$; ' $\mathrm{Of} 88$ healthy Ashkenazi Jewish patients subsequently tested, seven (7.95\%) were carriers of the mutation P369S. 
within the general population. The carrier rates were calculated after excluding positively identified homozygotes or compound heterozygotes (see below). The mutation M694V was the most common in the North African Jews, while in the Ashkenazi, Iranian and Iraqi groups, the mutation E148Q was found to be by far the most prevalent. Overall, the mutation E148Q was found to be the most frequent -0.07 (95\% Cl $=0.05-0.08)$. In Iraqi Jews it was as high as 0.115 $(95 \% \mathrm{Cl}=0.07-0.17)$, with frequencies of $0.075(95 \%$ $\mathrm{Cl}=0.04-0.08) \quad$ in Ashkenazi Jews, $0.05 \quad(95 \%$ $\mathrm{Cl}=0.02-0.09)$ in North African Jews, and $0.025(95 \%$ $\mathrm{Cl}=0.01-0.06)$ in Iranian Jews. Following in frequency was the mutation M694V, with an overall frequency of 0.035 (95\% Cl $=0.024-0.05)$, and as high as $0.08 \quad(95 \%$ $\mathrm{Cl}=0.05-0.13$ ) in Jews from North Africa (Table 1 ).

Table2 depicts the distribution of the mutations found in FMF patients. In North African Jews and I raqi Jews the most common mutation is M694V, and in Ashkenazi Jews the mutation V726A is the most prevalent. Mutation E148Q is only second or third in frequency in all groups.

Table3 compares the distribution of the different mutations between heal thy carriers and FMF patients ( $P<0.003)$. The commonest mutation in patients, M694V, was found in $84.4 \%$ of all patients from all ethnic groups, but in only $29 \%$ of carriers. In contrast, mutation E148Q was very frequent (53\%) among healthy carriers, but was found in only $6.6 \%$ of FMF patients. This difference was found in each ethnic group when analysed separately.

In the healthy population group used for the carrier screening study, we identified six adults who each carried two mutations. In the Iraqi group we found one individual who was homozygous for E148Q (aged 30), one compound heterozygote E148Q/M 694V (aged 30), and one compound heterozygote E148Q/V726A (aged 44). In the North African group we found one individual who was homozygous for E148Q (aged 26), and two individuals who were compound heterozygotes E148Q/M694V (aged29,30). On enquiry, none of these individuals had any signs or symptoms of FMF attacks or amyloidosis. Among the FMF patients no E148Q homozygotes were found, but there were patients with $\mathrm{E} 148 \mathrm{Q} / \mathrm{V} 726 \mathrm{~A}$ and $\mathrm{E} 148 \mathrm{Q} / \mathrm{M} 694 \mathrm{~V}$.

\section{Discussion}

This study demonstrates a high prevalence of the different MEFV mutations in all ethnic groups in Israel. This rate exceeds that expected by the prevalence of the disease, especially in Ashkenazi and Iraqi Jews, and therefore suggests that many patients in these ethnic groups are undiagnosed. Indeed, the carrier rate was previously estimated, based on family studies, to be as high as 1:5-1:7 among some nonAshkenazi Jewish populations, ${ }^{16}$ and 1:7 among Armenians in California. ${ }^{17}$ Owing to the high prevalence in Jews, Israeli doctors find themselves including FMF in their differential diagnosis quite often, especially when the patient is of North African origin. The carrier rates that we have found exceed these estimations, and to the best of our knowledge demonstrate the highest carrier rates for an autosomal recessive disease described so far. The discrepancy between the estimation of the carrier rates on the basis of family clinical studies and those found in this study is even greater for Iraqi Jews (39\%), and Ashkenazi Jews (22\%). This latter is in agreement with the carrier frequency of $21 \%$ recently found in Ashkenazi Jews. ${ }^{14}$ Thus these data may suggest a greater degree of clinical underdiagnosis, or of non-penetrance, or both, among Ashkenazi and Iraqi Jews. Indeed, the analysis of the different distributions of the mutations between the Jewish groups - Ashkenazi and Iraqi Jews have a higher prevalence of E148Q, whereas the North African Jews have a higher prevalence of M694V - might explain this phenomenon. Mutation E148Q was proportionately more common among the carriers than among the patients, further suggesting that this mutation may be associated with a high non-penetrance rate. We did, in fact, identify two individuals homozygous for E148Q and another four compound heterozygotes with E148Q who had no clinical evidence of inflammatory attacks or amyloidosis by age 26-44 years.

After we had completed the testing of the subjects described in this paper, we performed additional testing on Ashkenazi Jews for the presence of the mutation P369S. Out of 88 healthy individuals, seven were found to carry this mutation $(7.95 \%)$, whereas out of 58FMF patients, only two were found to be compound heterozygotes for this and

Table 2 Distribution of the MEFV mutations among FMF patients in different Jewish groups in Israel (mutation frequency for the known mutations)

\begin{tabular}{|c|c|c|c|c|c|c|c|c|}
\hline & M694V & V726A & M680I & E148Q & M694I & A744S & K695R & Unknown \\
\hline$M A J^{a}$ & $\begin{array}{l}205 \\
(94.4 \%)\end{array}$ & $\begin{array}{l}2 \\
(0.9 \%)\end{array}$ & - & $\begin{array}{l}9 \\
(4.1 \%)\end{array}$ & - & - & $\begin{array}{l}1 \\
(0.5 \%)\end{array}$ & 23 alleles \\
\hline Iraqi Jews & $\begin{array}{l}17 \\
(55 \%)\end{array}$ & $\begin{array}{l}8 \\
(26 \%)\end{array}$ & - & $\begin{array}{l}5 \\
(16 \%)\end{array}$ & - & $\begin{array}{l}1 \\
(3.2 \%)\end{array}$ & - & 9 alleles \\
\hline Other non-Ashkenazi Jews & $\begin{array}{c}13 \\
(65 \%)\end{array}$ & $\begin{array}{c}6 \\
(30 \%)\end{array}$ & - & $\begin{array}{l}1 \\
(5 \%)\end{array}$ & - & - & - & 43 alleles \\
\hline Ashkenazi Jews ${ }^{b}$ & $\begin{array}{l}10 \\
(38.5 \%)\end{array}$ & $\begin{array}{l}10 \\
(38.5 \%)\end{array}$ & - & $\begin{array}{l}4 \\
(15.3 \%)\end{array}$ & - & $\begin{array}{l}2 \\
(7.7 \%)\end{array}$ & - & 8 alleles \\
\hline
\end{tabular}

${ }^{\mathrm{a}} \mathrm{NAJ}=$ North African Jews; ${ }^{\mathrm{b}}$ Of 58 Ashkenazi Jewish patients subsequently tested, one (1.72\%) carried the mutation P369S. 
Table 3 Comparison of the distribution of the MEFV mutations found in FMF patients and in healthy individuals

\begin{tabular}{lclll}
\hline & M694V & V726A & M680I & E148Q \\
\hline FMF patients & $245(84.4 \%)$ & $26(9.0 \%)$ & - & $19(6.6 \%)$ \\
$\begin{array}{l}\text { (290 alleles) } \\
\text { Asymptomatic }\end{array}$ & $29(29 \%)$ & $16(16 \%)$ & $2(2 \%)$ & $53(53 \%)$ \\
adults with & & & & \\
$\begin{array}{l}\text { MEFV mutations } \\
\text { (100 alleles) }\end{array}$ & & & & \\
\hline
\end{tabular}

$\mathrm{P} \varangle 0.003$

E148Q (our data). This would suggest that P369S is probably another mild mutation which is common in Ashkenazi Jews, but further studies would be required to confirm this.

Previous studies have demonstrated a significant association between M694V and amyloidosis. ${ }^{6-11}$ Conversely, in our extensive series of FMF patients with amyloidosis, none of the patients who carried the mutation E148Q developed this complication. These findings concur with those of other researchers. ${ }^{6,10}$ There have been no documented cases of amyloidosis in homozygotes for E148Q and only one in a patient who was a compound heterozygote M694V/E148Q, ${ }^{18}$ which suggests that this mutation carries a relatively low risk for FMF related amyloidosis. So far, it would appear that amyloidosis may develop rarely only when this mutation is combined with M694V (ie M 694V/E148Q).

Our findings may have important clinical implications for the treatment of FMF families. Since colchicine has been shown to prevent the development of amyloidosis, ${ }^{2}$ lifelong treatment is recommended for all FMF patients. Based on our findings, it appears that homozygotes for M694V are at the highest risk, and should be given colchicine treatment for life. In mildly affected patients (those with infrequent inflammatory attacks) who do not carry this mutation, further studies are needed in order to determine whether the patients should be either treated, or tested every 6 months for the presence of proteinuria. This is particularly the case for those patients where one or both mutations are E148Q.

The high prevalence of mutation M694V in North African Jews (gene frequency of $0.08[95 \% \mathrm{Cl}=0.05-0.13]$ and carrier rate of 0.14 [ $95 \% \mathrm{Cl}=0.09-0.19]$ ), raises the question of the need for screening the newborns in this population, especially when both parents are from the same ethnic origin. Amyloidosis in such patients may appear in untreated individuals even prior to the onset of inflammatory attacks

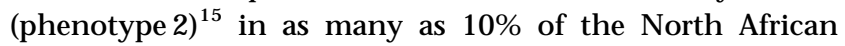
Jewish patients. Since this is preventable by colchicine therapy, it appears that end-stage renal failure could be prevented in 1:2000screened individuals. If further confirmed, screening for the mutation M694V among the North African Jewish population in Israel may appear to be justified, and should be considered for other at-risk groups in the future.

\section{Acknowledgements}

The authors thank theFMF families for participating in the study. This project was partially supported by a research award from Mifal Hapayis, by the Israeli Ministry of Health Chief Scientist grant No.4209, and by grant32/97 18.0 from the Israeli Academy of Science.

\section{References}

1 Sohar E, Gafni J, Pras M, Heller H: Familial Mediterranean fever. A survey of 470 cases and review of the literature. Am J Med 1967; 43: 227-253.

2 Livneh A, Zemer D, Langevitz ZP et al: Colchicine treatment of AA amyloidosis in familial Mediterranean fever. Arthritis Rheum 1994; 37: 1804-1811.

3 The International FMF Consortium: Ancient missense mutations in a new member of the RoRet gene family are likely to cause familial Mediterranean fever. Cell 1997; 90: 797-807.

4 The French FMF Consortium: A candidate gene for familial Mediterranean fever. Nat Genet 1997; 17: 25-31.

5 Bernot A, da Silva C, Petit JL et al: Non-founder mutations in the MEFV gene establish this gene as the cause of familial Mediterranean fever. Hum Mol Genet 1998; 7(8): 1317-1325.

6 Shohat M, Magal N, Shohat T et al: Phenotype-genotype correlation in familial Mediterranean fever: evidence for an association between Met694Val and amyloidosis. Eur J Hum Genet 1999; 7: 287-292.

7 Dewalle M, Domingo C, Rozen baum M et al: Phenotype-genotype correlation in Jewish patients suffering from familial Mediterranean fever (FMF). Eur J Hum Genet 1998; 6: 95-97.

8 Yalçinkaya F, Akar N, Misirlioglu M: Familial Mediterranean fever - amyloidosis and the Val726Ala mutation. (Letter). N EngJ Med 1998; 338: 993.

9 Pras M: Familial Mediterranean fever: From the clinical syndrome to the cloning of the pyrin gene. Scand J Rheumatol 1998; 27: 92-97.

10 Livneh A, Langevitz $P$, Shinar $Y$ et al: MEFV mutation analysis in patients suffering from amyloidosis in familial Mediterranean fever. Amyloid 1999; 6(1): 1-6.

11 Cazeneuve C, Sarkisian T, Pêcheux C et al: MEFV-gene analysis in Armenian patients with familial Mediterranean fever: Diagnostic value and unfavorable renal prognosis of the M $694 \mathrm{~V}$ homozygous genotype - genetic and therapeutic implications. Am J Hum Genet 1999; 65: 88-97.

12 Eliakim M, Rachmilewitz M, Rosenmann E et al: Renal manifestations in recurrent polyserositis (familial Mediterranean fever). Isr J Med Sci 1970; 6: 228-245.

13 Sohar E, Gafni J, Pras M et al: Familial Mediterranean fever. A survey of 470 cases and review of the literature. Am J Med 1967; 43: 227-253.

14 Aksentijevich I, Torosyan Y, Samuels J et al: Mutation and haplotype studies of familial Mediterranean fever reveal new ancestral relationships and evidence for a high frequency with reduced penetrance in the Ashkenazi Jewish population. Am J Hum Genet 1999; 64: 949-962.

15 Heller H, Sohar E, Gafni J, Heller J: Amyloidosis in familial Mediterranean fever. An independent genetically determined characteristic. Arch Intern Med 1961; 107: 539-550.

16 Daniels M, Shohat T, Brenner-Ullman A, Shohat M: Familial Mediterranean fever: high gene frequency among the nonAshkenazic and Ashkenazic Jewish populations in Israel. Am J M ed Genet 1995; 55(3): 311-314.

17 Rogers DB, Shohat M, Petersen GM et al: Familial Mediterranean fever in Armenians; autosomal recessive inheritance with high gene frequency. Am J Med Genet 1989; 34: 168-172.

18 Samuels J, Aksentijevich I, Torosyan $Y$ et al: Familial Mediterranean fever at the millenium. Clinical spectrum, ancient mutations, and a survey of 100 American referrals to the National Institutes of Health. Medicine 1998; 77: 268-297. 